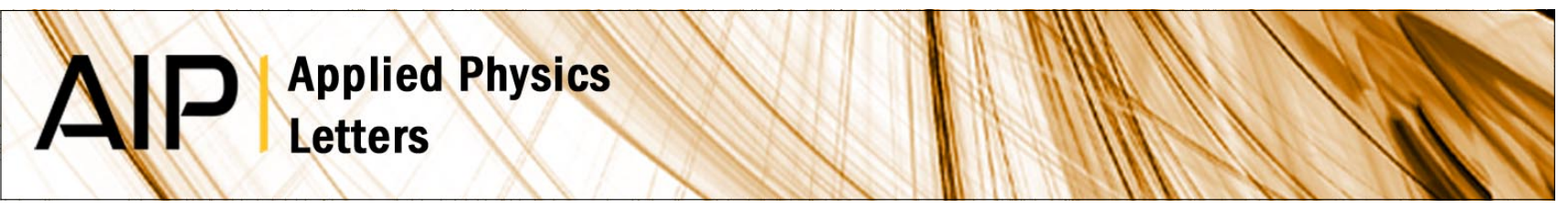

\title{
Energy-filtered phase retrieval using the transport of intensity equation
}

\author{
V. J. Keast, M. J. Gladys, T. C. Petersen, C. Dwyer, C. T. Koch et al.
}

Citation: Appl. Phys. Lett. 99, 221905 (2011); doi: 10.1063/1.3663572

View online: http://dx.doi.org/10.1063/1.3663572

View Table of Contents: http://apl.aip.org/resource/1/APPLAB/v99/i22

Published by the American Institute of Physics.

\section{Related Articles}

Direct observation of guided-mode interference in polymer-loaded plasmonic waveguide Appl. Phys. Lett. 101, 171116 (2012)

Stress evaluation in thin strained-Si film by polarized Raman spectroscopy using localized surface plasmon resonance

Appl. Phys. Lett. 101, 172101 (2012)

Ultrasmall radial polarizer array based on patterned plasmonic nanoslits

Appl. Phys. Lett. 101, 161119 (2012)

Localized surface plasmon resonances in highly doped semiconductors nanostructures

Appl. Phys. Lett. 101, 161113 (2012)

Polarization dependant scattering as a tool to retrieve the buried phase information of surface plasmon polaritons Appl. Phys. Lett. 101, 161603 (2012)

\section{Additional information on Appl. Phys. Lett.}

Journal Homepage: http://apl.aip.org/

Journal Information: http://apl.aip.org/about/about_the_journal

Top downloads: http://apl.aip.org/features/most_downloaded

Information for Authors: http://apl.aip.org/authors

\section{ADVERTISEMENT}
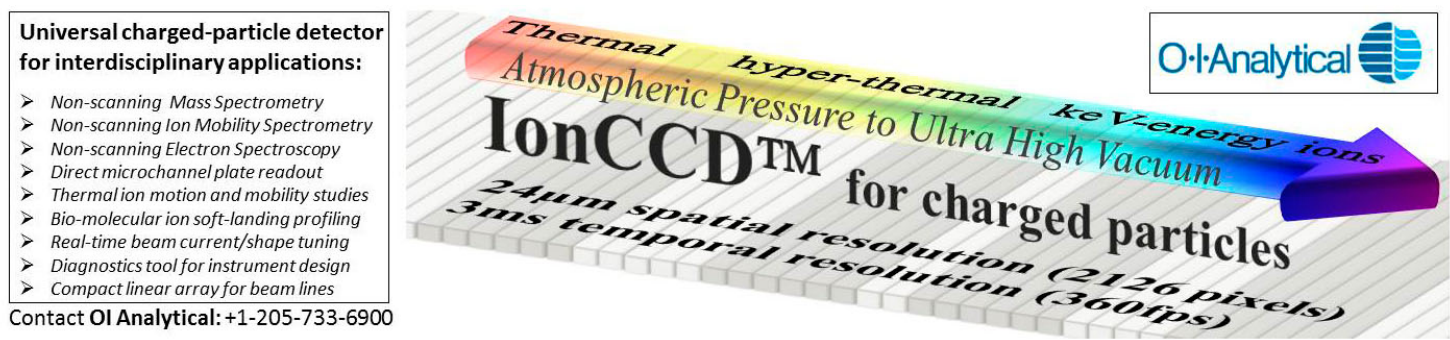


\title{
Energy-filtered phase retrieval using the transport of intensity equation
}

\author{
V. J. Keast, ${ }^{1}$ M. J. Gladys, ${ }^{1}$ T. C. Petersen, ${ }^{2}$ C. Dwyer, ${ }^{3}$ C. T. Koch, ${ }^{4}$ T. Haber, ${ }^{5}$ \\ and G. Kothleitner \\ ${ }^{1}$ School of Mathematical and Physical Sciences, The University of Newcastle, Callaghan, \\ New South Wales 2308, Australia \\ ${ }^{2}$ School of Physics, Monash University, Clayton, Victoria 3800, Australia \\ ${ }^{3}$ Monash Centre for Electron Microscopy, Department of Materials Engineering, and ARC Centre \\ of Excellence for Design in Light Metals, Monash University, Clayton, Victoria 3800, Australia \\ ${ }^{4}$ Department of Physics, Ulm University, 89081 Ulm, Germany \\ ${ }^{5}$ Institute for Electron Microscopy, Steyrergasse 17, 8010 Graz, Austria
}

(Received 4 October 2011; accepted 3 November 2011; published online 29 November 2011)

\begin{abstract}
Energy-filtered imaging was combined with phase retrieval to study plasmon scattering contributions to electron phase shifts surrounding gold nanoparticles. Spectral windows with sufficient energy resolution to exclude plasmon scattering were used to measure through-focal intensity derivatives and retrieve phase maps via the transport of intensity equation. Phase excursions in the phase maps that included plasmon scattering were found to disagree with theory by at least an order of magnitude. This discrepancy was attributed to subtle particle instabilities under the electron beam, which lead to systematic errors in the retrieved phase maps. (C) 2011 American Institute of Physics. [doi:10.1063/1.3663572]
\end{abstract}

In recent years, there has been renewed interest in surface plasmons on metallic nanoparticles for a diverse range of applications. ${ }^{1-3}$ Fundamental to progress in this field is the correlation of the local geometry of the nanostructure with the surface plasmon energy. Mapping surface plasmons at the nanoscale with energy filtering in the transmission electron microscope (EFTEM) or spectrum imaging (SI) in the scanning TEM (STEM) have been very successful in this endeavour. ${ }^{4-7}$ The energy resolution necessary to measure the surface plasmon requires the use of a TEM or STEM with a highly monochromatic electron source such as those found in cold field emission gun instruments or those with monochromators.

In earlier work, we measured electron phase shifts from metal nanoparticles using phase retrieval in the TEM. ${ }^{8}$ Unexpected deviations in the phase profiles at the edges of metal particles were interpreted to likely be associated with the excitation of surface plasmons. However, further work revealed that the measured phase change of $0.5-1.5 \mathrm{rad}$ from particle edges to surrounding vacuum exceeds theoretical prediction by at least an order of magnitude. For example, the phase change of $200 \mathrm{keV}$ electrons passing at grazing incidence to a $20 \mathrm{~nm}$ gold sphere is predicted to be $34 \mathrm{mrad}$. These calculations utilized the angle of deflection as predicted by classical electrodynamics (see also the related calculations by Anstis $^{9}$ ). Interestingly, earlier studies have also reported that the measured deflection of a focussed electron beam passing just external to a surface is also an order of magnitude larger than the classical prediction. ${ }^{10,11}$

In this work, we test whether measured electron phase shifts can be experimentally ascribed to plasmon losses for gold nano-particles, to better understand the discrepancy with theory. To this end, we have combined EFTEM with through-focal intensity measurements to perform energyfiltered phase retrieval.

The transport of intensity equation (TIE) provides one approach to retrieve electron phase shifts from through-focal intensity measurements. ${ }^{12-14}$ The TIE connects the intensity and phase to the intensity derivative in the direction of propagation. Using periodic boundary conditions, the TIE can be rapidly solved using fast Fourier transforms. ${ }^{15}$ A throughfocal image series can be used to calculate the intensity derivative in the focal plane by fitting a polynomial for the focal variation. This procedure has previously been shown to give a quantitative and robust measurement for the phase. $^{16,17}$

Surface plasmon excitations on gold nanostructures have energies at or below $2.5 \mathrm{eV}$, so that the separation of these inelastic contributions requires a monochromated TEM and a high resolution imaging filter with a very narrow energy-selecting slit. Our measurements were performed on two different TEMs: an optimised Tecnai F20, $200 \mathrm{kV}$ TEM fitted with a Wien filter monochromator ${ }^{18}$ and a Zeiss SESAM $200 \mathrm{kV}$ TEM equipped with an in-column energy filter ${ }^{19}$ and an electrostatic monochromator. ${ }^{20}$ Initially, the Tecnai F20 was equipped with a specially optimised highresolution Gatan imaging filter $(\mathrm{GIF})^{21}$ and in later experiments with a GIF Quantum. ${ }^{22}$

Energy-filtered through-focal images were acquired in two different ways. In the first approach, an energy-selecting slit was inserted and the through-focal series was acquired. Images containing only the elastic signal were obtained by using a slit small enough to exclude the plasmon excitations. The inelastic excitations were then included using a larger slit, still centred about zero, but large enough that the plasmon excitations also contributed to the image intensity. The smallest usable slit width was limited by the chromatic properties of the spectrometer; a slit that is too narrow produces substantial intensity variations across the field of view.

In the second approach, a small energy-selecting slit was chosen and a full EFTEM image series was acquired at each focal step to create an EFTEM-SI. The energy step size in the EFTEM-SI was smaller than the slit width and energy resolutions of $0.3-1 \mathrm{eV}$ were obtained. Each EFTEM series was first spatially realigned using the routine described in 
Schaffer et al $^{23}$ From the EFTEM-SIs elastic, plasmon and combined intensities were extracted through simple window integrals. More detailed spectral information was measured with this second approach, at the practical expense of comparatively fewer focal steps.

Both approaches yield a through-focal series of images where the intensity contributing to each image in the series originates from electrons with a specified range of energy losses. Sample drift between images at different focal steps was corrected by a manual realignment of the images. Realignment is necessary for phase retrieval but introduces slight shifts in the isochromatic pattern across the image series. The small defocus increments used mean that image rotation and magnification changes with defocus were not significant. Phase retrieval using the TIE was performed using methods described in previous work. ${ }^{17}$

Many data sets were acquired and processed of which two illustrative examples will be shown here. Fig. 1 shows an in-focus image, intensity derivative, and the retrieved phase images from a gold particle, acquired using the Tecnai F20 and HR-GIF. A $0.9 \mathrm{eV}$ wide energy-selecting slit was used to obtain Figs. 1(a), 1(c), and 1(e) and a $10 \mathrm{eV}$ energyselecting slit for Figs. 1(b), 1(d), and 1(f). The focal series consisted of 11 images separated by defocus increments of $50 \mathrm{~nm}$ (nominal). The intensity variation was fitted with a 2nd order polynomial, and the TIE was solved with no band pass filtering or regularisation.

Consistent with previous work, a broad phase excursion is seen surrounding the gold for the larger energy-selecting slit (Fig. 1(f)). The fact that no such profile is seen to surround the phase map for the small slit (Fig. 1(f)) would seem to suggest the effect is indeed due to plasmon scattering. However, further scrutiny of our experimental data has shown that such an interpretation is erroneous.

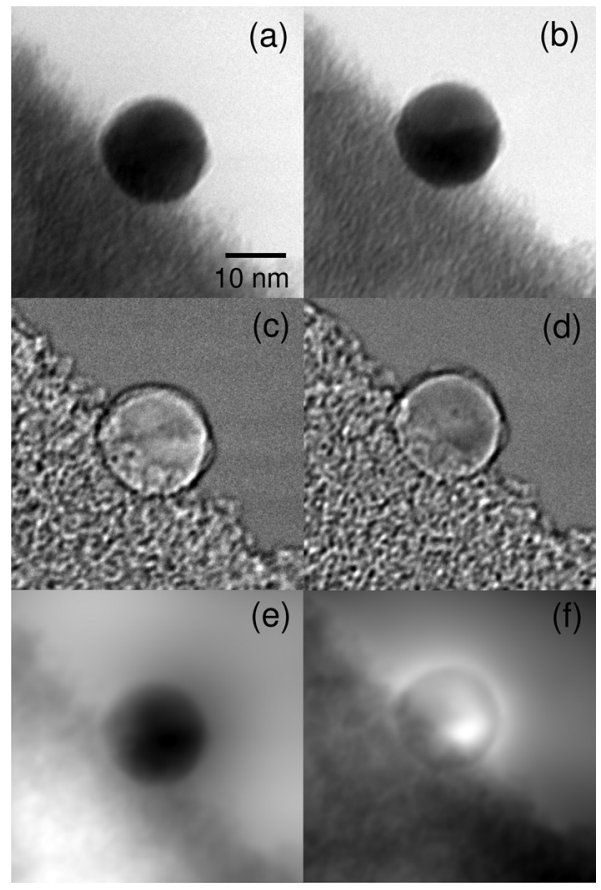

FIG. 1. (a) and (b) In-focus image, (c) and (d) intensity derivative, and (e) and (f) retrieved phase images from a gold particle. A $0.9 \mathrm{eV}$ wide energyselecting slit was used to obtain (a), (c), and (e) and a $10 \mathrm{eV}$ energyselecting slit for (b), (d), and (f).
Consider Fig. 2, which shows an in-focus image, intensity derivative, and retrieved phase images from a set of gold nanoparticles. This data were acquired using the Zeiss SESAM and the second approach with a $0.23 \mathrm{eV}$ monochromator slit and a $1.3 \mathrm{eV}$ energy-selecting slit. Using nominal defocus increments of $50 \mathrm{~nm}, 17$ through focal images were collected. Some residual astigmatism remained uncorrected in each image. Through-focal intensity variations were fitted with a 3rd order polynomial, and a high-pass filter was applied to the intensity derivative to remove apparent lowspatial frequency artefacts. The data in Fig. 2 clearly show one gold particle displaying broad phase excursions, whilst adjacent particles do not.

Analysis of many more data sets identified that diminished values of the intensity derivative inside the particle can produce a halo in the phase outside the particle. This is demonstrated in Fig. 2 where the topmost particle shows a broad dip in the intensity derivative inside the bottom section. The corresponding phase map portrays a halo around the particle, as well as a deviation inside. Close examination also reveals that the intensity derivative inside the particle is slightly lower in Fig. 1(d) than in Fig. 1(c).

Fig. 3 shows a simulation demonstrating these effects. For the intensity derivative Fig. 3(b), the middle of the circular region was set slightly lower than that for Fig. 3(a). Using a uniform intensity and these simulated derivatives, the TIE was solved to give Figs. 3(c) and 3(d). The phase maps are strikingly different, and the halo is clearly apparent. This effect demonstrates how the non-local nature of this approach to phase retrieval necessitates particular care in the interpretation of data; the phase determined at any particular

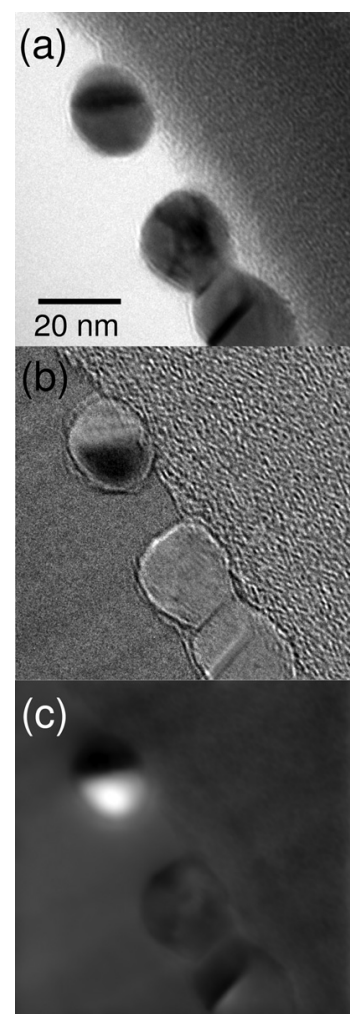

FIG. 2. (a) In-focus image, (b) intensity derivative, and (c) retrieved phase images from gold particles. In this instance, only one particle displays phase deviations. These occur at a region of a particle with the intensity derivative has a noticeably lower value. 


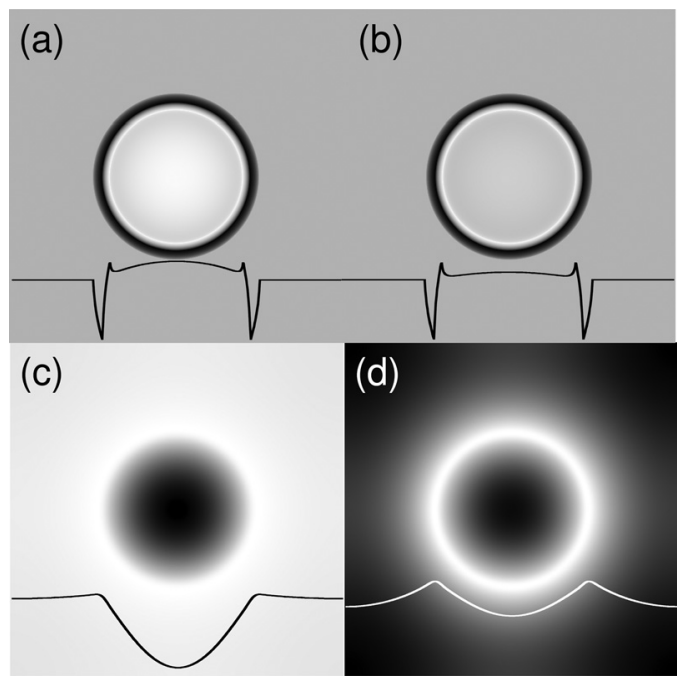

FIG. 3. (a) and (b) Maps and profiles of an intensity derivative artificially constructed to look similar to those observed experimentally. In (b), the intensity derivative in the middle of the circular region is lower than in (a). The corresponding retrieved phase is shown in (c) and (d).

point will be influenced by the intensity derivative in other regions.

The phenomenological reason for the broad dip in the intensity derivative of Fig. 2 was revealed by visually examining the experimental through focal images (not shown here). The intensity was observed to vary subtly but nonmonotonically with defocus-appearing to flicker upon scanning through focus. One physical explanation is that this part of the particle was close to a Bragg condition, producing strong diffraction contrast, which is quite sensitive to changes in crystal orientation. In general, these small metal particles are not stable under the beam. Examination of the focal series associated with Fig. 1 did not display any obvious effects such as these, and a number of other data sets showing the phase deviations also did not. However, the possibility that a similar, but even more subtle, effect is occurring cannot be excluded. Other unusual phase effects in metal nanoparticles have been observed elsewhere ${ }^{24}$ and may have a similar origin. In fact, since all through focal experiments assume that intensity variations are solely due defocus aberrations rather than erratic, time-dependent, phenomena, such phase retrieval errors are not unique to the TIE.

To avoid the fluctuating diffraction contrast described above, stable particles that maintain their precise crystal orientation during data acquisition are required. Experimentally, better stability can be achieved by embedding the particles in a dielectric matrix and/or by imaging at lower beam intensities. Single crystal particles or amorphous specimens would also enable better control of diffraction contrast.

When considering systematic errors for the TIE, some consistency tests can be applied, such as the requirement that the intensity derivative should effectively average to zero using conservation of energy. ${ }^{25}$ Fresnel propagation can also be used to check the consistency of the phase solution with the experimental through focal images. However, for the data used to create Fig. 1, such checks could not distinguish between the two phase maps within the measurement uncertainties.
In summary, we have demonstrated two different methods for performing energy filtered phase retrieval with energy resolutions below $1 \mathrm{eV}$. Experiments designed to separate contributions to the phase from plasmon scattering and from elastic scattering could not differentiate these signals on account of particle instabilities and associated diffraction contrast fluctuations. This work has shown that erroneous phase excursions surrounding gold nano-particles can arise from such artefacts, which explains a discrepancy with theoretical expectations of this interesting phenomenon. Substantial improvements could be made by embedding specimens in a dielectric matrix and/ or by imaging at lower operating voltages. Specimens with higher plasmon energy, such as aluminium particles, would also reduce demands on the spectroscopy.

B. Schaffer and W. Grogger are thanked for providing the EFTEM alignment script. B. Kraus of Gatan is thanked for assistance on modifying the GIF quantum quadrupole settings for improved energy resolution. T.C.P. would like to acknowledge D. M. Paganin and J. Barry for useful comments. Financial support was provided by the Australian Research Council (ARC) and additional travel support by the Deutscher Akademischer Austauschdienst (DAAD). C.T.K. acknowledges funding from the Carl Zeiss Foundation.

${ }^{1}$ J. Homola, S. S. Yee, and G. Gauglitz, Sens. Actuators B 54, 3 (1999).

${ }^{2}$ S. A. Maier and H. A. Atwater, J. Appl. Phys. 98, 011101 (2005).

${ }^{3}$ K. A. Willets and R. P. Van Duyne, Annu. Rev. Phys. Chem. 58, 267 (2007).

${ }^{4}$ M. Bosman, V. J. Keast, M. Watanabe, A. I. Maaroof, and M. B. Cortie, Nanotechnology 18, 165505 (2007).

${ }^{5}$ J. Nelayah, M. Kociak, O. Stéphan, F. J. García de Abajo, M. Tencé,

L. Henrard, D. Taverna, I. Pastoriza-Santos, L. M. Liz-Marzán, and C. Colliex, Nat. Phys. 3, 348 (2007).

${ }^{6}$ B. Schaffer, U. Hohenester, A. Trugler, and F. Hofer, Phys. Rev. B 79 (2009). ${ }^{7}$ W. Sigle, J. Nelayah, C. T. Koch, B. Ogut, L. Gu, and P. A. Van Aken, Ultramicroscopy 110, 1094 (2010).

${ }^{8}$ T. C. Petersen, M. Bosman, V. J. Keast, and G. R. Anstis, Appl. Phys. Lett. 93, 101909 (2008).

${ }^{9} \mathrm{G}$. Anstis, Ultramicroscopy 110, 1070 (2010).

${ }^{10} \mathrm{P}$. M. Echenique and A. Howie, Ultramicroscopy 16, 269 (1985).

${ }^{11}$ C. S. Tan and J. M. Cowley, Ultramicroscopy 12, 333 (1984).

${ }^{12}$ S. Bajt, A. Barty, K. A. Nugent, M. McCartney, M. Wall, and D. Paganin, Ultramicroscopy 83, 67 (2000).

${ }^{13}$ K. Ishizuka and B. Allman, J. Electron Microsc. 54, 191 (2005).

${ }^{14}$ M. R. Teague, J. Opt. Soc. Am. 73, 1434 (1983).

${ }^{15}$ D. Paganin and K. A. Nugent, Phys. Rev. Lett. 80, 2586 (1998).

${ }^{16}$ T. C. Petersen, V. J. Keast, K. Johnson, and S. Duvall, Philos. Mag. 87, 3565 (2007).

${ }^{17}$ T. C. Petersen, V. J. Keast, and D. M. Paganin, Ultramicroscopy 108, 805 (2008).

${ }^{18}$ D. S. Su, H. W. Zandbergen, P. C. Tiemeijer, G. Kothleitner, M. Havecker, C. Herbert, A. Knop-Gericke, B. H. Freitag, F. Hofer, and R. Schlogl, Micron 34, 235 (2003).

${ }^{19}$ E. Essers, G. Benner, T. Mandler, S. Meyer, D. Mittmann, M. Schnell, and R. Höschen, Ultramicroscopy 110, 971 (2010).

${ }^{20}$ C. T. Koch, W. Sigle, R. Höschen, M. Rühle, E. Essers, G. Benner, and M. Matijevic, Microsc. Microanal. 12, 506 (2006).

${ }^{21}$ G. Kothleitner and F. Hofer, Micron 34, 211 (2003).

${ }^{22}$ A. Gubbens, M. Barfels, C. Trevor, R. Twesten, P. Mooney, P. Thomas, N. Menon, B. Kraus, C. Mao, and B. McGinn, Ultramicroscopy 110, 962 (2010).

${ }^{23}$ B. Schaffer, W. Grogger, and G. Kothleitner, Ultramicroscopy 102, 27 (2004).

${ }^{24}$ P. Donnadieu, S. Lazar, G. A. Botton, I. Pignot-Paintrand, M. Reynolds, and S. Perez, Appl. Phys. Lett. 94, 263116 (2009).

${ }^{25}$ T. E. Gureyev, A. Roberts, and K. A. Nugent, J. Opt. Soc. Am. A 12, 1932 (1995). 